\title{
GWAS in people of Middle Eastern descent reveals a locus protective of kidney function-a cross-sectional study
}

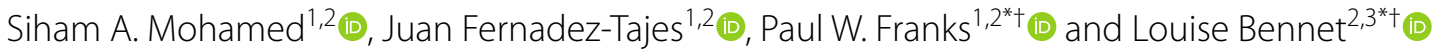

\begin{abstract}
Background: Type 2 diabetes is one of the leading causes of chronic kidney failure, which increases globally and represents a significant threat to public health. People from the Middle East represent one of the largest immigrant groups in Europe today. Despite poor glucose regulation and high risk for early-onset insulin-deficient type 2 diabetes, they have better kidney function and lower rates of all-cause and cardiovascular-specific mortality compared with people of European ancestry. Here, we assessed the genetic basis of estimated glomerular filtration rate (eGFR) and other metabolic traits in people of Iraqi ancestry living in southern Sweden.

Methods: Genome-wide association study (GWAS) analyses were performed in 1201 Iraqi-born residents of the city of Malmö for eGFR and ten other metabolic traits using linear mixed-models to account for family structure.

Results: The strongest association signal was detected for eGFR in CST9 (rs13037490; $P$ value $=2.4 \times 10^{-13}$ ), a locus previously associated with cystatin C-based eGFR; importantly, the effect (major) allele here contrasts the effect (minor) allele in other populations, suggesting favorable selection at this locus. Additional novel genome-wide significant loci for eGFR (ERBB4), fasting glucose (CAMTA1, NDUFA10, TRIO, WWC1, TRAPPC9, SH3GL2, ABCC11), quantitative insulin-sensitivity check index (METTL16), and HbA1C (CAMTA1, ME1, PAK1, RORA) were identified.
\end{abstract}

Conclusions: The genetic effects discovered here may help explain why people from the Middle East have better kidney function than those of European descent. Genetic predisposition to preserved kidney function may also underlie the observed survival benefits in Middle Eastern immigrants with type 2 diabetes.

Keywords: Genome-wide study, Chronic kidney disease, Estimated glomerular filtration rate, Middle East, Type 2 diabetes

\section{Background}

Chronic kidney disease (CKD) is a leading public health problem, affecting more than $13 \%$ of the world's population [1]. Along with aging, obesity, and hypertension, type 2 diabetes (T2D) is one of the leading causes of CKD

*Correspondence: paul.franks@med.lu.se; louise.bennet@med.lu.se ${ }^{\dagger}$ Paul W. Franks and Louise Bennet contributed equally to this work. ${ }^{2}$ Department of Clinical Sciences, Lund University, Malmö, Sweden

${ }^{3}$ Clinical Research and Trial Center, Lund University Hospital, Lund, Sweden

Full list of author information is available at the end of the article
[2], with 1:10 deaths in people with diabetes attributed to kidney failure [3].

CKD is caused by a gradual loss of kidney function characterized by diminished glomerular filtration rate (GFR) and/or other markers of kidney damage [4]. Variants in genes like UMOD, GPX1, GSTO1, GSTO2, SHROOM3, and MGP have been associated with susceptibility to CKD [5]. The prevalence of CKD varies between ethnicities, with African Americans at particularly high risk $[6,7]$. 
The city of Malmö is multicultural, hosting people from approximately 180 countries. Every third citizen is born abroad, with the largest immigrant group born in Iraq and representing $3.4 \%$ of the total population [8]. The MEDIM cohort (the impact of Migration and Ethnicity on Diabetes in Malmö) is a population-based cohort comprised of people aged 30 to 75 years, born in either Iraq or Sweden, and resident in Malmö. Data from the MEDIM study has revealed that Iraqi migrants in Malmö are at twice the risk of T2D [9] and have younger age of disease onset compared with the Swedish born population, with high burden of diabetes family history, and poorer glycemic control and insulin sensitivity [10]. Paradoxically, despite the poorer metabolic health profile, Iraqi migrants present with better kidney function and lower blood pressure than their Swedish counterparts [11], rendering this a particularly interesting cohort for genetic studies of these traits.

In the last decade, GWAS has improved the understanding of the genetic architecture of polygenic diseases such as CKD and T2D [12]. However, these studies were performed predominantly in European ancestry populations, which may limit generalizability of findings to other ethnicities, underscoring the need for greater ethnic diversity in GWAS [13]. Regardless of trait, no published GWAS has been performed in cohorts of Iraqi ancestry.

The purpose of this study was to undertake GWAS of eGFR and 10 additional diabetes-related traits in immigrants of Iraqi ancestry from the MEDIM cohort.

\section{Methods}

\section{Study participants and phenotyping}

The phenotyping process has been described in detail previously $[9,14]$. Briefly, after signing informed consent, participants aged 30 to 75 years were randomly selected from the Malmö census register. Individuals with severe physical or mental illness were excluded. The final cohort included here comprised 1201 men and women born in Iraq with complete GWAS data (see Additional file 1: Fig S1).

Assessments were undertaken from February 1, 2010, through December 31, 2012. Participants were invited to a health exam and were told not to eat or drink anything besides water and not to utilize tobacco from $10 \mathrm{pm}$ the day preceding testing; they were also asked to record their current medication. A standard physical checkup was performed prior to sample collection. Clinical variables such as waist circumference, height, weight, body mass index (BMI), systolic blood pressure (SBP), and diastolic blood pressure (DBP) were assessed. DNA for genotyping was extracted from buffy coat. Blood samples were collected when participants were fasting and during a 75-g oral glucose tolerance test (OGTT) from which insulin sensitivity index (ISI), corrected insulin response (CIR), and oral disposition index (DIO) were calculated [15]. Blood glucose, homeostasis model assessment of $\beta$ cell function (HOMA- $\beta$ ), glycated hemoglobin (HbA1C), quantitative insulin sensitivity check index (QUICKI), and estimated glomerular filtration rate (eGFR) were assessed in fasting samples. eGFR was calculated based on the mean of eGFR creatinine and eGFR cystatin C. A detailed overview of how eGFR was calculated is provided elsewhere [11]. QUICKI and HOMA- $\beta$ were computed as follows:

$$
\begin{aligned}
& \text { QUICKI }=1 /[\log (F I)+\log (F G)] \\
& H O M A-\beta=20 \times F I / F G-3.5,
\end{aligned}
$$

where FG and FI denotes fasting glucose $(\mathrm{mmol} / \mathrm{L})$ and fasting insulin (mIE/L) respectively.

\section{Statistics}

\section{Genotyping and quality control}

Genotyping was performed at the Swedish National Genomics Infrastructure - SciLifeLab (Uppsala, Sweden) using the Infinium assay (Illumina, USA), and data were analyzed using GenomeStudio 2.0.3 (Illumina, USA) [16]. The genome build used for genotype curation was 37 (GRCh37). A total of 759,993 SNP markers were analyzed with a genotyping call rate of $99.26 \%$.

Phenotype data were log-transformed to approximate a normal distribution. SNPs and individuals with low genotype calls were excluded using a threshold of 0.02 . Sex discrepancy was checked based on X chromosome heterozygosity, and, where necessary, sex was determined using genotype. SNPs with a minor allele frequency (MAF) $<0.01$ were removed owing to insufficient samples size, as were SNPs deviating from Hardy-Weinberg equilibrium $\left(p\right.$ value $\left.<1 \times 10^{-6}\right)$. Individuals with a heterozygosity rate deviating more than $3 \mathrm{SD}$ from the sample mean were also excluded. Duplicate individuals and cryptic relatedness among samples were checked using PLINK's $\pi$-hat pairwise identity by descent (IBD) estimate, calculated as follows:

$$
\pi-h a t=\mathrm{P}(I B D=2)+0.5 \times \mathrm{P}(I B D=1)
$$

where $P$ represented probability

A threshold of $\pi$-hat $>0.2$ was used to classify pairs as being related. Duplicate individuals (22 individuals) with a $\pi$-hat $=1$ were removed. A total of 1201 individuals and 482959 SNPs survived quality control (Additional file 1: Fig S1).

Population stratification was checked using the multidimensional scaling (MDS) approach. To check for 
possible outliers, an MDS plot was generated anchored to the 1000 Genomes dataset of known ethnic background. The first seven MEDIM MDS were retained and used as covariates for the association analysis. The standard quality control steps mentioned above and control for population stratification was performed using PLINK version 1.9 (an open-source whole genome association analysis toolset), and R-version 4.1.0 was used for generating plots.

\section{Imputation}

The quality-controlled genotype data was prepared for imputation using the Haplotype Reference Consortium (HRC) preparation checking tool. Imputation was performed via Michigan Imputation Server (www. imputationserver.sph.umich.edu) executed by minimac4 algorithm and European HRC panel. Phasing was implemented by Eagle v2.4. Following imputation, SNPs were filtered based on imputation info $\left(R^{2} \geq 0.3\right)$ and MAF $>0.01$.

\section{Association analysis}

The associations between the 7,743,666 genotyped and imputed SNPs and the 11 quantitative traits were analyzed using linear mixed models. The 11 traits were fasting glucose, HOMA- $\beta$, HbA1C, BMI, CIR, ISI, DIO, QUICKI, SBP, DBP, and eGFR. Age, sex, and the first seven MDS principal components were included as covariates. The association analyses were conducted using BOLT-LMM software [17]. By default, BOLT-LMM assumes a Bayesian mixture-of-normal prior for the random effect attributed to SNPs other than the one being tested. This random effect reflects the polygene background and environmental effect that could affect the calculation of genetic association. Example of these random effects could be cryptic relatedness or population structure.

A conventional genome-wide significance threshold of $P<5.0 \times 10^{-8}$ was used. After the association analysis, significant SNPs were clumped to determine the lead representative SNP within a 250-kb LD block. Clumping was done using the integrative web-based platform, FUMA (https://fuma.ctglab.nl/) [18]. The variant effect for the lead SNP was predicted using Ensembl Variant Effect Predictor (VEP) [19]. Results from this analysis were cross-referenced with the NHGRI-EBI GWAS Cata$\log$ to identify previously reported signals [20].

\section{Replicates}

Not applicable

\section{Key resources}

The following RRIDs tools and dataset were utilized in the GWAS:

Functional Mapping and Annotation of Genome Wide Association Studies, RRID:SCR_017521

PLINK, RRID:SCR_001757

R Project for Statistical Computing, RRID:SCR_001905

Michigan Imputation Server, RRID:SCR_017579

GWAS: Catalog of Published Genome-Wide Association Studies, RRID:SCR_012745

1000 Genomes: A Deep Catalog of Human Genetic Variation, RRID:SCR 006828

SAMtools/BCFtools, RRID:SCR_005227

\section{Results}

A European reference panel was used for imputation owing to the unavailability of Arab-ancestry haplotype reference panels. Of the available ancestral reference panels, the MEDIM cohort aligns most closely with the European-ancestry reference panel (Additional file 1: Fig S2).

In analyses assessing the degree of relatedness among MEDIM participants, 431 individual pairs with a $\pi$-hat value $>0.2$ were found. Out of these individuals, 22 pairs were identified as duplicate individuals. For each duplicate pair, the observation with the lowest genotyping call rate was removed. In a histogram, the number of related MEDIM participants is presented (Additional file 1: Fig S3).

The mean age of the MEDIM cohort was 46.2 years, and the majority was male $(60.5 \%)$. The average eGFR in the study population was $89.8 \mathrm{ml} / \mathrm{min}$ per $1.73 \mathrm{~m}^{2}$. The characteristics of study participants are shown in Additional file 2: Table S1.

After performing the GWAS analyses, 19 loci were significantly $\left(P\right.$ value $\left.<5 \times 10^{-8}\right)$ associated with fasting glucose, HbA1c, QUICKI, and eGFR (see Fig. 1 and Additional file 1: Fig S15-S32). The list of lead SNP is given in Table 1. eGFR had the most genome-wide significant SNP associations, with 107 significant SNPs within chromosome 20 (see Fig. 2), all showing an increasing effect of the minor allele on eGFR. The strongest association among these variants was seen for rs 13037490 $\left(P\right.$ value $=2.4 \times 10^{-13}$, see Fig. 2$)$, a $3^{\prime}$ UTR variant localizing to CST9 in a previously reported locus [21]. For the same trait, we found an additional independent genome-wide significant variant $\left(P\right.$ value $\left.=4.6 \times 10^{-08}\right)$, an intronic variant in $E R B B 4$ (see Additional file 1: Fig. S26). Nominal $\left(1 \times 10^{-7}>P<0.05\right)$ signals for four traits (fasting glucose, ISI, CIR, and HOMA-B) in MEDIM 

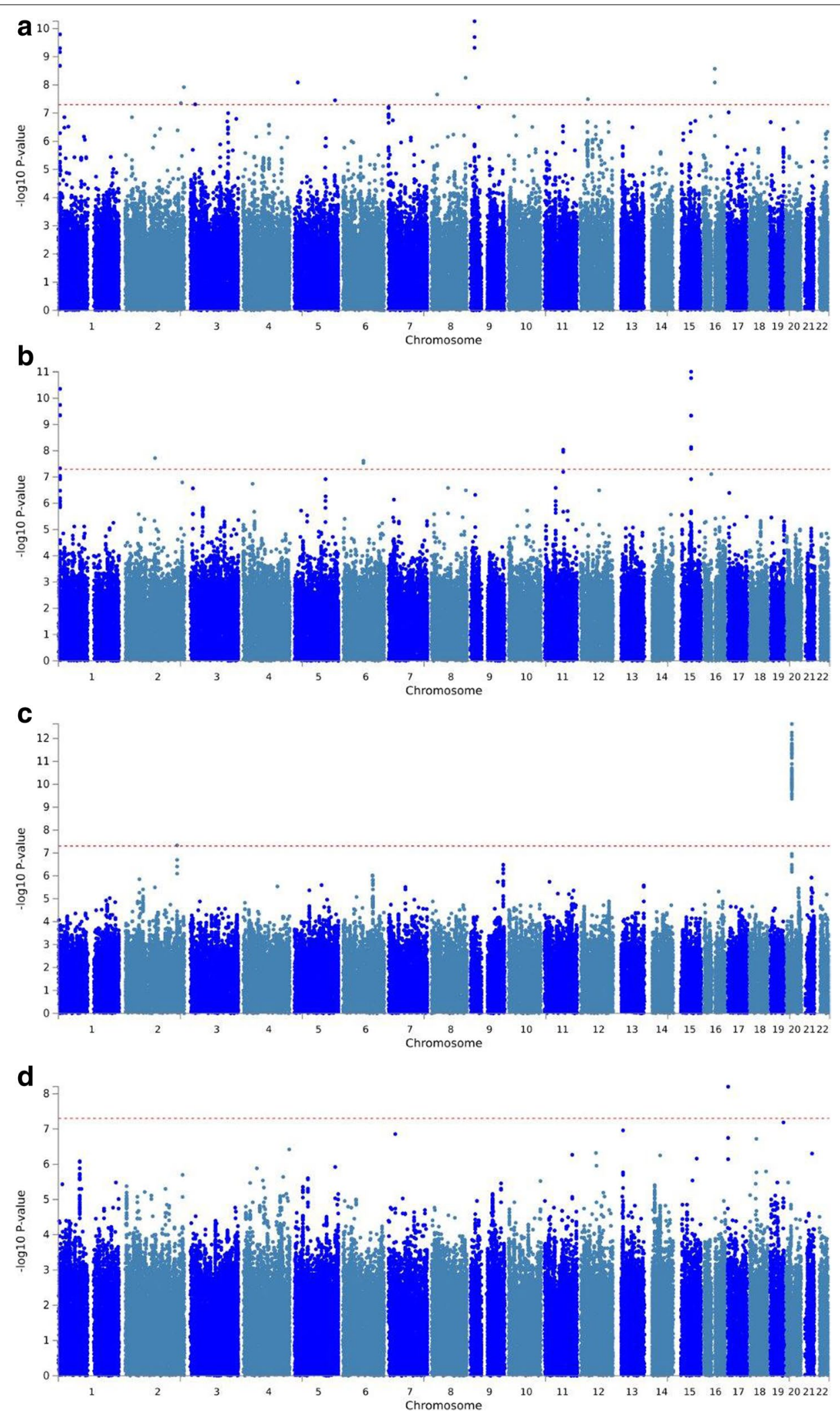

Fig. 1 Manhattan plots for the association of SNPs with A fasting glucose, B HbA1C, C eGFR, and D QUICKI. Graph displays -log $10 P$ values ( $y$-axis) against chromosomal location ( $x$-axis). The red line indicates genome-wide significance threshold $\left(P\right.$ value $\left.=5 \times 10^{-8}\right)$ 
Table 1 Lead SNPs (with genome-wide significant signals) associated with FG, HbA1C, QUICKI, and eGFR

\begin{tabular}{|c|c|c|c|c|c|c|c|c|}
\hline Trait & Lead SNPs & Gene/nearest gene & Chr & Position $^{a}$ & Effect allele & Beta & SE & $P$ value \\
\hline eGFR & rs73985808 & ERBB4 & 2 & 212565932 & A & -0.08 & 0.01 & $4.6 \times 10^{-08}$ \\
\hline eGFR & rs13037490 & CST9 & 20 & 23583725 & C & 0.08 & 0.01 & $2.4 \times 10^{-13}$ \\
\hline $\mathrm{FG}$ & rs11120828 & CAMTA1 & 1 & 7122846 & A & 0.14 & 0.02 & $1.6 \times 10^{-10}$ \\
\hline FG & rs76150693 & & 2 & 228526473 & G & 0.33 & 0.042 & $4.4 \times 10^{-08}$ \\
\hline FG & rs79451541 & NDUFA10 & 2 & 240929777 & A & 1.03 & 0.18 & $1.2 \times 10^{-08}$ \\
\hline FG & rs74591871 & & 3 & 20241645 & A & 0.196 & 0.036 & $4.9 \times 10^{-08}$ \\
\hline FG & rs78223279 & TRIO & 5 & 14397518 & A & 0.68 & 0.19 & $8.3 \times 10^{-09}$ \\
\hline$F G$ & rs115873798 & WWC1 & 5 & 167759448 & G & 0.25 & 0.04 & $3.4 \times 10^{-08}$ \\
\hline$F G$ & rs73231408 & & 8 & 24930722 & $\mathrm{~T}$ & 0.75 & 0.12 & $2.2 \times 10^{-08}$ \\
\hline FG & rs147360587 & TRAPPC9 & 8 & 141360595 & C & 0.23 & 0.04 & $5.6 \times 10^{-09}$ \\
\hline$F G$ & rs143653828 & SH3GL2 & 9 & 17580151 & $\mathrm{~T}$ & 0.31 & 0.05 & $5.4 \times 10^{-11}$ \\
\hline$F G$ & rs77023105 & & 12 & 30658931 & G & 0.33 & 0.06 & $3.2 \times 10^{-08}$ \\
\hline$F G$ & rs72802149 & $A B C C 11$ & 16 & 48197315 & $\mathrm{~T}$ & 0.6 & 0.1 & $2.7 \times 10^{-09}$ \\
\hline $\mathrm{HbA} 1 \mathrm{C}$ & rs11120828 & CAMTA1 & 1 & 7122846 & A & 0.16 & 0.02 & $4.4 \times 10^{-11}$ \\
\hline $\mathrm{HbA} 1 \mathrm{C}$ & rs77145902 & & 2 & 121862595 & $\mathrm{~T}$ & 0.28 & 0.05 & $1.9 \times 10^{-08}$ \\
\hline $\mathrm{HbA} 1 \mathrm{C}$ & rs117580692 & ME1 & 6 & 83955807 & A & 1.45 & 0.25 & $2.4 \times 10^{-08}$ \\
\hline $\mathrm{HbA} 1 \mathrm{C}$ & rs72941612 & PAK1 & 11 & 77059870 & $\mathrm{~T}$ & 0.32 & 0.05 & $9.1 \times 10^{-09}$ \\
\hline $\mathrm{HbA} 1 \mathrm{C}$ & rs146006303 & $R O R A$ & 15 & 61137932 & C & 1.13 & 0.16 & $9.7 \times 10^{-12}$ \\
\hline QUICKI & rs184544915 & METTL16 & 17 & 2329349 & C & 0.49 & 0.08 & $6.3 \times 10^{-09}$ \\
\hline
\end{tabular}

a Position is according to Build 37 (GRCh37/hg19)

were listed as "highly ranked" variants in GWAS Catalog, suggesting that these signals are likely to be false negative in MEDIM owing to insufficient statistical power (see Additional file 2: Table S2). Quantile-quantile (Q-Q) plots illustrating significantly associated traits are shown in Additional file 1: Fig S33 for eGFR, HbA1c, fasting glucose, and QUICKI.

Several steps were undertaken to characterize the functional basis of rs13037490 (CST9) and rs73985808 (ERBB4). We began by examining variants within the LD block $\left(r^{2}>0.8\right.$, determined from the 1000 Genomes Project - internationalgenome.org) within which rs13037490 and rs73985808 reside, using HaploReg V4.1 software (HaploReg v4.1 (broadinstitute.org). Here, the functional basis of linked SNPs and small indels is ascertained using (i) annotations from Roadmap Epigenomics and ENCODE projects, (ii) sequence conservation across mammals, (iii) effect of SNPs on regulatory motifs, and (iv) the effect of index SNPs on gene transcription. Variants in high LD with rs13037490 are characterized by a variety of functional properties, with no clearly discernible pattern of causality. Thus, to narrow the search space further, we used CAUSALdb Index (http://www. mulinlab.org) to identify a $95 \%$ credible causal set, which revealed five likely causal variants, all in high LD with our index SNP rs13037490 (Additional file 2, Table S3). Because rs13037490 was in LD with this credible set but did not rank above these five variants (13th rank), it is likely that rs13037490 is not causal in and of itself but is a strong tag for the causal locus. However, the functional annotation suggests that the causal effects are likely to be primarily in testis and blood, with no clear indication of kidney-specific effects. For rs73985808, there was little evidence of function, other than that rs73985808 disrupts the binding motifs for FOXL1, FOXP1, and PAX-4. We also explored the possibility of using the MEDIM data for direct annotations using GARFIELD (https://www. ebi.ac.uk/birney-srv/GARFIELD/), but determined from the low estimates of certainty that the MEDIM dataset is likely to be underpowered for this purpose (data not shown), and elected not to proceed with further analyses of this nature.

\section{Discussion}

This is one of the first analyses investigating genetic variants associated with kidney function and T2D in a Middle Eastern ancestry cohort and the first GWAS to be reported for any trait in a cohort of Iraqi ancestry. We identified a genome-wide significant signal at CST9 for eGFR, which has also been detected in other ethnicities [21, 22]. CST9 encodes a secreted protein believed to play a role in hematopoietic differentiation and inflammation. Variation at CST9 has also previously been linked with cystatin C [23]. In European-ancestry populations, CST9 variants rs1158167 $\left(P\right.$ value $\left.=8.5 \times 10^{-09}\right)$ and $\operatorname{rs} 214523146\left(P\right.$ value $\left.=1.1 \times 10^{-05}\right)[24]$ have also 


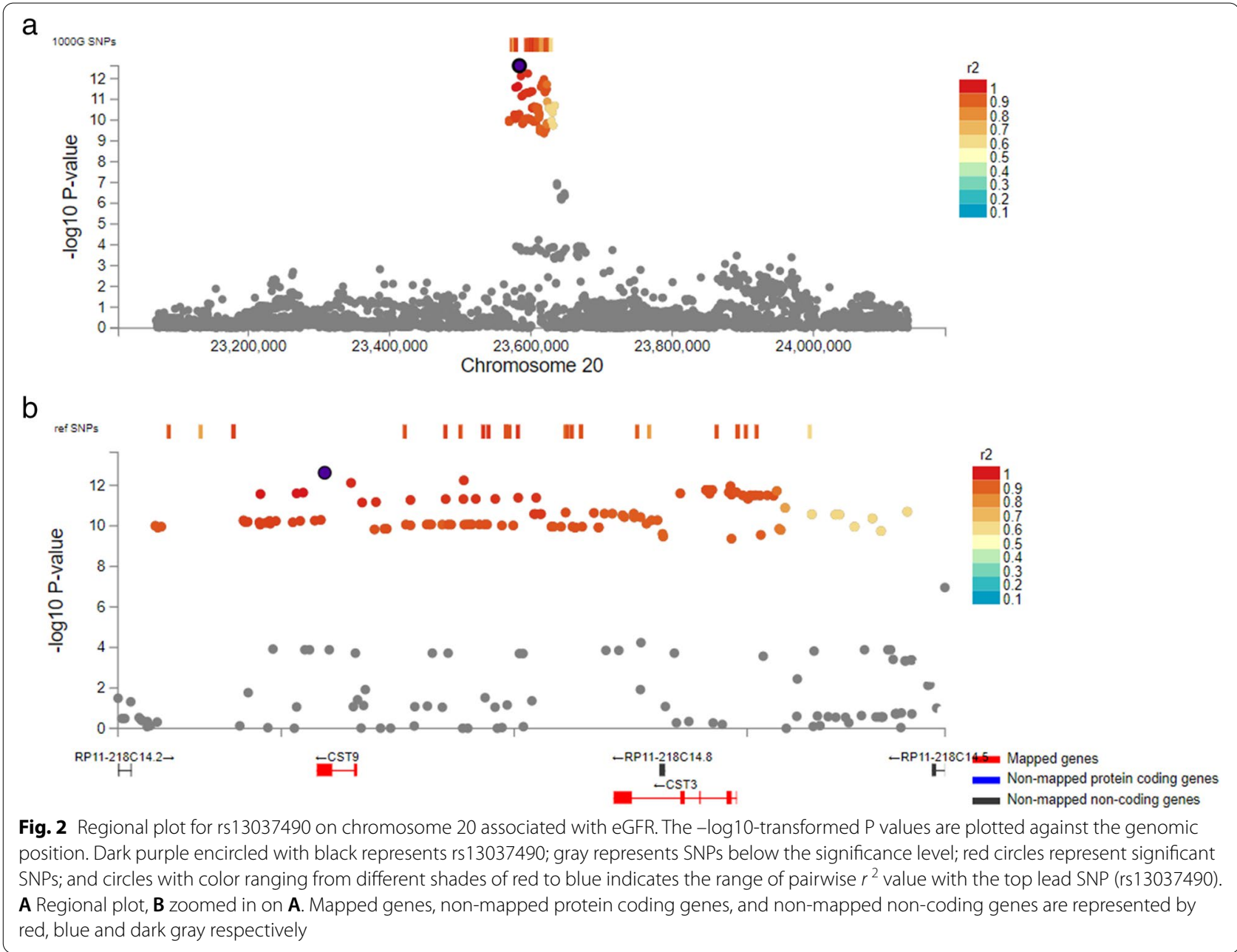

previously been associated with serum cystatin $C$ levels, as has rs1303830545 $\left(P\right.$ value $\left.=2.2 \times 10^{-88}\right)[21]$ with eGFRcys.

A second key finding from this study concerns variants at $E R B B 4$ and kidney function. ERBB4 is a member of the EGF receptor (EGFR) subfamily of receptor tyrosine kinases and plays a critical role developing epithelial ducts in the kidney [25]. A variant in ERBB4 has been previously associated with diabetic nephropathy [26], and ERBB4/Erbb4 has been shown to be differently expressed in human in vitro and in murine models of renal disease [25]. The negative beta for ERBB4 and eGFR shows that the minor allele is associated with lower eGFR (i.e., worse kidney function). Thus, the major allele (i.e., the majority of the MEDIM population) carries the protective allele. This contrasts observations in other ethnic groups, where the risk allele is the most prevalent (major) allele. It is for this reason that we conclude the Iraqi population may be genetically protected from chronic kidney disease. A previous study focusing on renal function and its association with blood pressure in MEDIM participants reported that this Iraqi cohort had better overall kidney function than native Swedes [11]. Elsewhere, a Swedish nationwide study of people with new onset T2D reported that first-generation non-Western immigrants, and Middle Eastern immigrants in particular, have lower rates of allcause and cause-specific mortality than ancestral Swedes with new onset diabetes [27]. The current analysis indicates that the better kidney function in Iraqis compared with native Swedes may have a genetic basis, with ERBB4 being a key locus.

The most obvious explanation for the signals observed here and not in large European ancestry GWAS cohorts is that the genetic architecture of these cohorts differs, at least at the index loci. A second explanation is that the genetic signals may be a consequence of gene-environment interactions, early life environments (intrauterine and years $1-5$ postpartum) being possible examples [28]. 
A limitation of this analysis is the lack of an Iraqi (or ethnically-proximal) replication cohort, which is not currently available. The importance of using a Middle Eastern cohort for replication studies can be observed when trying to replicate the strongest common variant signals for T2D. For example, rs7903146 and rs12255372, localizing to TCF7L2, have been widely replicated across many ethnic groups including American Indians [29], Japanese [30], South Asians [31, 32], Pakistanis [33], Afro-Caribbeans [32], and Europeans, but not in Middle Eastern cohorts [34]. Indeed, barely a handful of loci identified in European ancestry populations for metabolic traits have been replicated in Middle Eastern cohorts [35-37]. The genetic discordance between Middle Eastern and non-Middle Eastern cohorts, observed here for the ERBB4 signals, further underscores the importance of replication studies being performed in cohorts from the Middle East.

Nevertheless, in the absence of an ethnically proximal replication cohort, we looked up the strongest signals from the current analysis in GWAS Catalog (European ancestry cohorts) to determine if any published findings exist that support those reported here. Although this process has its limitations, most notably that GWAS Catalog only includes genome-wide significant results and does not include data from Middle Eastern cohorts, some of our strongest findings correspond well with those reported there. We also used the Type 2 Diabetes Knowledge Portal [38] to determine if any of our strongest hits are associated with any sub-genome-wide significant signals in European-ancestry cohorts, which we also found some evidence of, suggesting that the Iraqi population, and the features of its genome, may be more powerful for genetic discovery than European ancestry cohorts, at least for kidney function traits.

There are currently no publicly available imputation panels for people of Iraqi ancestry. When comparing patterns of genetic variation in this Iraqi cohort with other ethnicities for whom reference panels are available, it was determined that the most proximal of these was the European ancestry panel. While this is unlikely to be adequate for imputation of rare variants, for common variant imputation, which is the focus of this paper, it yields acceptable predictions of missing variants. Regardless, the region where the strongest signals were detected in this study includes $>100$ variants, the majority of which were directly genotyped. In this case, imputation has no bearing on the reliability of the signal, as the signal is primarily driven by non-imputed SNPs.

Even though the signals detected for the glycemic traits are not accompanied by replication studies, most of the mapped genes had literature evidence for their involvement in the pathogenesis of T2D or glucose metabolism. For instance, CAMTA1 was associated with T2D in a French population [39]. Several studies highlight the involvement of CAMTA1 in the development of mature functional cells of islets as well as in regulating beta-cell insulin content and secretion. NDUFA10 codes for the enzyme $42 \mathrm{kDa}$ complex I and is involved in oxidative phosphorylation (OXPHOS) inside the mitochondria. This gene, along with other OXPHOS genes, was found to be downregulated in pancreatic islets of T2D patients and was implicated in the development of impaired glucose-stimulated insulin secretion [40, 41]. TRIO (Rho guanine nucleotide exchange factor), which was associated with fasting glucose, plays a role in cell migration and growth through the actin cytoskeleton's reorganization. Dufurrena et al. recently demonstrated TRIO's active role in the regulation of glucose responsiveness and proinsulin secretion [42]. SH3GL2 (SH3 Domain Containing GRB2 Like 2, Endophilin A1) is known for its active role in lipid binding and lipid tube assembly. In 2012, SH3GL2 was added to the list of a candidate genes for T2D, which might affect islet function [43]. $A B C C 11$ codes for $\mathrm{ABC}$ proteins, which transport molecules across extra- and intra-cellular membranes, including glucose and other sugars. A variant in $A B C C 11$ (ATP binding cassette subfamily $C$ member 11 ) gene was associated with fasting glucose in a meta-analysis of 13 genome-wide association studies [44]. ME1 codes for the cytosolic malic enzyme of pancreatic $\beta$ cells. $M E 1$ enzyme links the glycolytic and citric acid cycles. This gene is known to be highly expressed when dietary carbohydrate intake is elevated and is believed to actively enhance insulin secretion [45]; although its role in insulin secretion is contested [46]. PAK1 encodes for p21 (RAC1) activated kinase 1 proteins, which is an effector that connects the RhoGTPases to the cytoskeleton and nuclear signaling. PAK1 is involved in the second phase of glucose-stimulated insulin secretion [47] and Islets from individuals with T2D have been found deficient in PAK1 protein expression when compared with islets of individuals without diabetes $[48,49]$. RORA is a member of a nuclear hormone receptor that regulates gene expression. This gene regulates the transcription of genes, which are crucial for regulation of glucose metabolism. RORA has been identified as a transcriptional activator of insulin [50] and may impact T2D risk through numerous pathways [51].

Although the sample size used here is small relative to many contemporary GWAS, it is evidentially adequately powered to detect signals across multiple traits and is similar in size to early GWAS cohorts. Nevertheless, the nature of the MEDIM cohort requires the use of special statistical methods to account for cryptic relatedness and 
family structure, which may have diminished statistical power. Accordingly, it is likely that type 2 error (falsenegative rates) will be high in the current analysis, motivating future studies in larger Iraqi cohorts.

\section{Conclusion}

This is the first GWAS to be reported within an Iraqi population. Despite its relatively small sample size, we identified novel variants associated with kidney function, glycemic control, and insulin action. The apparent genetic protection from kidney dysfunction in this cohort may help explain why people from Iraqi appear to have better kidney function than people of European ancestry.

\begin{abstract}
Abbreviations
BMI : Body mass index; CKD: Chronic kidney disease; CIR: Corrected insulin response; DBP: Diastolic blood pressure; DIO: Oral disposition index; DNA: Deoxyribonucleic acid; eGFR : Estimated glomerulation rate; FG: Fasting glucose; GFR: Glomerulation rate; GWAS: Genome-wide association study; HbA1c: Glycated hemoglobin; HOMA- $\beta$ : Homeostasis model assessment, beta; HOMA-IR: Homeostasis model assessment, insulin resistance; HRC: Haplotype Reference Consortium; IBD: Identity by descent; ISI: Insulin sensitivity index; LMM: Linear mixed model; LUDC: Lund University Diabetes Center; MAF: Minor allele frequency; MDS: Multidimensional scaling; MEDIM: The impact of Migration and Ethnicity on Diabetes In Malmö; OGTT: Oral glucose tolerance test; Q-Q plot: Quantile-quantile plot; QUICKI : Quantitative insulin sensitivity check index; SBP: Systolic blood pressure; SNP: Single nucleotide polymorphism; T2D: Type 2 diabetes.
\end{abstract}

\section{Supplementary Information}

The online version contains supplementary material available at https://doi, org/10.1186/s12916-022-02267-7.

Additional file 1: Fig. S1. Flow chart for eligible study participants. Fig S2. Two-dimensional plots for multidimensional scaling of MEDIM participant and 1000 genomes populations. The distances between points reflect the genetic similarity. $Y$ axis $=2$ nd MDS component and $\mathrm{x}$ axis $=$ first MDS component. Fig S3. Histogram of pairwise genetic relatedness (PI-HAT) values calculated for all pairs in MEDIM participants. Fig. S4 - Fig. S14. Manhattan plot illustrating the $-\log 10$ ( $p$ values) from the MEDIM GWAS for all the 11 traits. Fig. S15- Fig. S32. Regional plot for all significantly associated Locus. Fig. S33. Quantile-quantile (Q-Q) plots of significantly association traits. Red line denotes null hypothesis $(X=Y)$, $\mathrm{x}$-axis $=$ observed $-\log 10[P] \& y$-axis $=$ Expected $\log 10[P] .(A)$ eGFR, (B) $\mathrm{HbA1C}$, (C) Fasting glucose and (D) QUICKI.

Additional file 2: Table S1. Demographic and clinical characteristics of the MEDIM participants. Table S2. Top 20 hit SNPs in GWAS catalog found in MEDIM GWAS. Table S3. Characterization of the functional basis of rs13037490 (CST9) assessing CAUSALdb Index (http://www.mulinlab.org) to identify a $95 \%$ credible causal set.

\section{Acknowledgements}

The authors express gratitude to the MEDIM Study participants for making this work possible. We also thank Gabriella Gremsperger at Biomedical Analytics at Wallenberg laboratory, Skåne University Hospital in Malmö for handling the storage of the blood samples, Mattias Borell and Johan Hultman for IT support at Lund University Diabetes Centre, and Tomas Axelsson and colleagues at the National Genomics Platform at SciLife, Uppsala, Sweden.

\section{Authors' contributions}

SAM and JFT analyzed the data; SAM and PWF wrote the article; JFT participated in writing and drafting the article and revising it critically; PWF contributed to the design of the work, data analyses and interpretation, in drafting the article and revising it critically; LB contributed to the conception and design of the work, in writing and drafting the article and revising it critically. The given sequence is the consensus of all contributors. All authors read and approved the final manuscript.

\section{Funding}

Open access funding provided by Lund University. Grants awarded that supported the work described in this paper: PWF: ERC-2015-CoG - 681742 NASCENT; Swedish Research Council (Distinguished Young Researcher Award in Medicine); Novo Nordisk Foundation. LB: Lund University (ALF grants 20101641, 20101837, and 162641), the Swedish Research Council (Linné grant to LUDC 349-2006-237, Exodiab 2009-1039, 2019-00978), the Swedish foundation for Strategic Research (LUDC IRC15-0067).

\section{Availability of data and materials}

Access to data will be determined on a case-by-case basis, after careful evaluation of whether a given data analysis proposal is consistent with the informed consent and ethics approval for the MEDIM study and whether it overlaps with any ongoing research in the MEDIM cohort.

\section{Declarations}

\section{Ethics approval and consent to participate}

The reported investigations have been carried out in accordance with the principles of the Declaration of Helsinki [52]. The study protocol was approved by the regional ethics review board in Lund, Sweden (\#2009/36; \#2010/561). All participants have provided written informed consent at enrollment into the study: Prior to signing the informed consent, they were informed about the study orally and in writing. They were also informed that they at any point could withdraw their participation in the study and withdraw their data from the database without explanation. All information and consent was provided in their spoken language.

\section{Consent for publication}

Not applicable since this study did not include any individual person's data.

\section{Competing interests}

The authors declare that they have no competing interests.

\section{Author details}

${ }^{1}$ Lund University Diabetes Center, Lund University, Malmö, Sweden. ${ }^{2}$ Department of Clinical Sciences, Lund University, Malmö, Sweden. ${ }^{3}$ Clinical Research and Trial Center, Lund University Hospital, Lund, Sweden.

Received: 28 September 2021 Accepted: 18 January 2022 Published online: 01 March 2022

\section{References}

1. LV JC, Zhang LX. Prevalence and disease burden of chronic kidney disease. Adv Exp Med Biol. 2019;1165:3-15.

2. Kazancioglu R. Risk factors for chronic kidney disease: an update. Kidney Int Suppl (2011). 2013;3(4):368-71.

3. van Dieren S, Beulens JW, van der Schouw YT, Grobbee DE, Neal B. The global burden of diabetes and its complications: an emerging pandemic. Eur J Cardiovasc Prev Rehabil. 2010;17(Suppl 1):S3-8.

4. Levey AS, Coresh J. Chronic kidney disease. Lancet. 2012;379(9811):165-80.

5. Corredor Z, Filho M, Rodriguez-Ribera L, Velazquez A, Hernandez A, Catalano C, et al. Genetic variants associated with chronic kidney disease in a Spanish population. Sci Rep. 2020;10(1):144.

6. Siemens TA, Riella MC, Moraes TP, Riella CV. APOL1 risk variants and kidney disease: what we know so far. J Bras Nefrol. 2018;40(4):388-402. 
7. Genovese G, Friedman DJ, Pollak MR. APOL1 variants and kidney disease in people of recent African ancestry. Nat Rev Nephrol. 2013;9(4):240-4.

8. Statistics Sweden. Available at http://www.scb.se [Internet]. Statistics Sweden. Available from: http://www.scb.se.

9. Bennet L, Groop L, Franks PW. Ethnic differences in the contribution of insulin action and secretion to type 2 diabetes in immigrants from the Middle East compared to native Swedes. Diabetes Res Clin Pract. 2014;105(1):79-87.

10. Bennet L, Franks PW, Zoller B, Groop L. Family history of diabetes and its relationship with insulin secretion and insulin sensitivity in Iraqi immigrants and native Swedes: a population-based cohort study. Acta Diabetol. 2018;55(3):233-42.

11. Nilsson C, Christensson A, Nilsson PM, Bennet L. Renal function and its association with blood pressure in Middle Eastern immigrants and native Swedes. J Hypertens. 2017;35(12):2493-500.

12. Pranavchand R, Reddy BM. Genomics era and complex disorders: implications of GWAS with special reference to coronary artery disease, type 2 diabetes mellitus, and cancers. J Postgrad Med. 2016;62(3):188-98.

13. Genetics for all. Nat Genet. 2019;51(4):579.

14. Bennet L, Johansson SE, Agardh CD, Groop L, Sundquist J, Rastam L, et al. High prevalence of type 2 diabetes in Iraqi and Swedish residents in a deprived Swedish neighbourhood - a population based study. BMC public health. 2011;11(1):303.

15. Matsuda M, DeFronzo RA. Insulin sensitivity indices obtained from oral glucose tolerance testing: comparison with the euglycemic insulin clamp. Diabetes Care. 1999;22(9):1462-70.

16. Convert array data into meaningful results. https://www.illumina.com/ techniques/microarrays/array-data-analysis-experimental-design/ genomestudio.html: Illumina; [

17. Loh PR, Tucker G, Bulik-Sullivan BK, Vilhjalmsson BJ, Finucane HK, Salem RM, et al. Efficient Bayesian mixed-model analysis increases association power in large cohorts. Nat Genet. 2015;47(3):284-90.

18. Watanabe K, Taskesen E, van Bochoven A, Posthuma D. Functional mapping and annotation of genetic associations with FUMA. Nat Commun. 2017:8(1):1826

19. McLaren W, Gil L, Hunt SE, Riat HS, Ritchie GR, Thormann A, et al. The Ensembl variant effect predictor. Genome Biol. 2016;17(1):122.

20. Buniello A, MacArthur JAL, Cerezo M, Harris LW, Hayhurst J, Malangone C, et al. The NHGRI-EBI GWAS Catalog of published genome-wide association studies, targeted arrays and summary statistics 2019. Nucleic Acids Res. 2019;47(D1):D1005-D12.

21. Kottgen A, Glazer NL, Dehghan A, Hwang SJ, Katz R, Li M, et al. Multiple loci associated with indices of renal function and chronic kidney disease. Nat Genet. 2009:41(6):712-7.

22. Gorski M, van der Most PJ, Teumer A, Chu AY, Li M, Mijatovic V, et al. 1000 Genomes-based meta-analysis identifies 10 novel loci for kidney function. Sci Rep. 2017;7:45040.

23. Witasp A, Ekstrom TJ, Lindholm B, Stenvinkel P, Schalling M, Nordfors $L$. Novel insights from genetic and epigenetic studies in understanding the complex uraemic phenotype. Nephrol Dial Transplant. 2014;29(5):964-71.

24. Hwang SJ, Yang Q, Meigs JB, Pearce EN, Fox CS. A genome-wide association for kidney function and endocrine-related traits in the NHLBI's Framingham Heart Study. BMC Med Genet. 2007;8 Suppl 1:S10.

25. Veikkolainen V, Naillat F, Railo A, Chi L, Manninen A, Hohenstein P, et al. ErbB4 modulates tubular cell polarity and lumen diameter during kidney development. J Am Soc Nephrol. 2012;23(1):112-22.

26. Sandholm N, Salem RM, McKnight AJ, Brennan EP, Forsblom C, Isakova T, et al. New susceptibility loci associated with kidney disease in type 1 diabetes. PLoS Genet. 2012;8(9):e1002921.

27. Bennet L, Udumyan R, Ostgren CJ, Rolandsson O, Jansson SPO, Wandell P. Mortality in first- and second-generation immigrants to Sweden diagnosed with type 2 diabetes: a 10 year nationwide cohort study. Diabetologia. 2021;64(1):95-108. https://doi.org/10.1007/s00125-02005279-1. Epub 2020 Sep 26. PMID: 32979073; PMCID: PMC7716891.

28. Franks PW, McCarthy MI. Exposing the exposures responsible for type 2 diabetes and obesity. Science. 2016;354(6308):69-73.

29. Chandak GR, Janipalli CS, Bhaskar S, Kulkarni SR, Mohankrishna P, Hattersley AT, et al. Common variants in the TCF7L2 gene are strongly associated with type 2 diabetes mellitus in the Indian population Diabetologia. 2007;50(1):63-7.

30. Hayashi T, Iwamoto Y, Kaku K, Hirose H, Maeda S. Replication study for the association of TCF7L2 with susceptibility to type 2 diabetes in a Japanese population. Diabetologia. 2007;50(5):980-4.

31. Humphries SE, Gable D, Cooper JA, Ireland H, Stephens JW, Hurel SJ, et al. Common variants in the TCF7L2 gene and predisposition to type 2 diabetes in UK European Whites, Indian Asians and Afro-Caribbean men and women. J Mol Med (Berl). 2006;84(12):1005-14.

32. Rees SD, Bellary S, Britten AC, O'Hare JP, Kumar S, Barnett AH, et al. Common variants of the TCF7L2 gene are associated with increased risk of type 2 diabetes mellitus in a UK-resident South Asian population. BMC Med Genet. 2008;9:8.

33. Melzer D, Murray A, Hurst AJ, Weedon MN, Bandinelli S, Corsi AM, et al. Effects of the diabetes linked TCF7L2 polymorphism in a representative older population. BMC Med. 2006;4:34

34. Alsmadi O, Al-Rubeaan K, Mohamed G, Alkayal F, Al-Saud H, Al-Saud NA, et al. Weak or no association of TCF7L2 variants with type 2 diabetes risk in an Arab population. BMC Med Genet. 2008;9:72.

35. Hebbar P, Abubaker JA, Abu-Farha M, Alsmadi O, Elkum N, Alkayal $F$, et al. Genome-wide landscape establishes novel association signals for metabolic traits in the Arab population. Hum Genet. 2021;140(3):505-28.

36. Hebbar P, Elkum N, Alkayal F, John SE, Thanaraj TA, Alsmadi O. Genetic risk variants for metabolic traits in Arab populations. Sci Rep. 2017;7:40988.

37. Ghassibe-Sabbagh M, Haber M, Salloum AK, Al-Sarraj Y, Akle Y, Hirbli $K$, et al. T2DM GWAS in the Lebanese population confirms the role of TCF7L2 and CDKAL1 in disease susceptibility. Sci Rep. 2014:4:7351.

38. Type 2 Diabetes Knowledge Portal hugeamp.org; [cited 2021 August 31st 2021]. Available from: https://t2d.hugeamp.org/.

39. Cauchi S, Proenca C, Choquet H, Gaget S, De Graeve F, Marre M, et al. Analysis of novel risk loci for type 2 diabetes in a general French population: the D.E.S.I.R. study. J Mol Med (Berl). 2008;86(3):341-8.

40. Olsson AH, Yang BT, Hall E, Taneera J, Salehi A, Nitert MD, et al. Decreased expression of genes involved in oxidative phosphorylation in human pancreatic islets from patients with type 2 diabetes. Eur J Endocrinol. 2011;165(4):589-95.

41. Wu C, Xu G, Tsai SA, Freed WJ, Lee CT. Transcriptional profiles of type 2 diabetes in human skeletal muscle reveal insulin resistance, metabolic defects, apoptosis, and molecular signatures of immune activation in response to infections. Biochem Biophys Res Commun. 2017:482(2):282-8

42. Dufurrena Q. Back N. Hodgson L, Tanowitz H, Mandela P, et al. Kalirin/ Trio Rho GDP/GTP exchange factors regulate proinsulin and insulin secretion. J Mol Endocrinol: Mains RE; 2018.

43. Taneera J, Lang S, Sharma A, Fadista J, Zhou Y, Ahlqvist E, et al. A systems genetics approach identifies genes and pathways for type 2 diabetes in human islets. Cell Metab. 2012;16(1):122-34.

44. Hwang JY, Sim X, Wu Y, Liang J, Tabara Y, Hu C, et al. Genome-wide association meta-analysis identifies novel variants associated with fasting plasma glucose in East Asians. Diabetes. 2015;64(1):291-8.

45. Heart E, Cline GW, Collis LP, Pongratz RL, Gray JP, Smith PJ. Role for malic enzyme, pyruvate carboxylation, and mitochondrial malate import in glucose-stimulated insulin secretion. Am J Physiol Endocrinol Metab. 2009:296(6):E1354-62.

46. Hasan NM, Longacre MJ, Stoker SW, Kendrick MA, MacDonald MJ. Mitochondrial malic enzyme 3 is important for insulin secretion in pancreatic beta-cells. Mol Endocrinol. 2015;29(3):396-410.

47. Veluthakal R, Chepurny OG, Leech CA, Schwede F, Holz GG, Thurmond DC. Restoration of glucose-stimulated Cdc42-Pak1 activation and insulin secretion by a selective epac activator in type 2 diabetic human islets. Diabetes. 2018;67(10):1999-2011.

48. Wang Z, Oh E, Clapp DW, Chernoff J, Thurmond DC. Inhibition or ablation of p21-activated kinase (PAK1) disrupts glucose homeostatic mechanisms in vivo. J Biol Chem. 2011;286(48):41359-67.

49. Kalwat MA, Yoder SM, Wang Z, Thurmond DC. A p21-activated kinase (PAK1) signaling cascade coordinately regulates F-actin remodeling and insulin granule exocytosis in pancreatic beta cells. Biochem Pharmacol. 2013;85(6):808-16. 
50. Kuang J, Hou X, Zhang J, Chen Y, Su Z. Identification of insulin as a novel retinoic acid receptor-related orphan receptor alpha target gene. FEBS Lett. 2014;588(6):1071-9.

51. Zhang Y, Liu Y, Liu Y, Zhang Y, Su Z. Genetic variants of retinoic acid receptor-related orphan receptor alpha determine susceptibility to type 2 diabetes mellitus in Han Chinese. Genes (Basel). 2016;7(8):54. https://doi.org/10.3390/genes7080054. PMID: 27556492; PMCID: PMC4999842.

52. WMA. Declaration of Helsinki - Ethical principles of medical research involving human subjects. 2008:http://www.wma.net/en/30publications/ 10policies/b3/index.html.

\section{Publisher's Note}

Springer Nature remains neutral with regard to jurisdictional claims in published maps and institutional affiliations.

- fast, convenient online submission

- thorough peer review by experienced researchers in your field

- rapid publication on acceptance

- support for research data, including large and complex data types

- gold Open Access which fosters wider collaboration and increased citations

- maximum visibility for your research: over $100 \mathrm{M}$ website views per year

At BMC, research is always in progress.

Learn more biomedcentral.com/submissions 